every 2 weeks. An MDT session on some of these cases was undertaken every 6 weeks.

Results 10 consultant delivered clinics were held. There were 41 new appointments with 3 DNAs and 7 follow ups with 2 DNAs. The DNA rate was $7.3 \%$ for new patient slots (hospital DNA rate $14.7 \%$ ) The follow up to new patient ratio was 1:1.03 (The ratio was 1: 1.56 ratio for the hospital based clinic). 2 patients were cancelled (4\%) as opposed to $18 \%$ cancellation rate in hospital clinics.

Overall, there was a reduction of $21 \%$ of all GP referrals (hub and non-hub clinics) in comparison to the same period in 2016. There was a reduction in referrals of $24 \%$ from the practice hosting the hub clinics.

In the opinion of the consultant doing the clinic, the majority of children could have been managed in primary care with GP education (53\%); GP advice and guidance $(71 \%)$ or by an alternative practitioner (53\%).

$96 \%$ of patients were positive about the booking process, the clinic and follow up arrangements.100\% of the MDT session evaluations were positive. The GPs fed back formally and informally that the resource was highly valued.

Conclusion The DNA rate and follow up to new ratio was favourable probably due to the short wait to see the consultant. Parents valued the service as it was close to home and in a familiar environment. GPs valued the MDT teaching sessions. Primary care services need to be supported by secondary care services to bring care closer to home.

\section{G82(P) HOW CONFIDENT ARE PAEDIATRICIANS AT MANAGING ADOLESCENTS WITH ACUTE MENTAL HEALTH PROBLEMS?}

${ }^{1} \mathrm{~A}$ Rajesh, ${ }^{2} \mathrm{~B}$ Alden, ${ }^{2} \mathrm{C}$ Kavanagh, ${ }^{3} \mathrm{~B}$ Fisher. ${ }^{1}$ Community Paediatrics, Norfolk Community Health and Care Trust, Norwich, UK; ${ }^{2} J e n n y$ Lind Childrens Hospital, Norfolk and Norwich University Hospital, Norwich, UK; ${ }^{3}$ Gurdon Institute, University of Cambridge, Cambridge, UK

\subsection{6/archdischild-2018-rcpch.80}

Background Mental health problems are increasing in prevalence within the paediatric population. Paediatricians are expected to work alongside CAMHS teams to assess and support these children, often with little training. In rare cases a child may require sectioning under the Mental Health Act.

This issue was previously raised in $\mathrm{RCPCH}$ consultation 'Looked after children: knowledge, skills and competences of health care staff'.

Aims We wanted to examine the experience and training of paediatricians in managing children with mental health problems, particularly with sectioning children.

Methods An online questionnaire was sent to paediatricians across one training deanery. Information was gathered on frequency and experience of sectioning children. We also looked at knowledge regarding available support and training.

Results 27 paediatricians responded: 13 (48\%) consultants, 12 $(44.5 \%)$ registrars, and $2(7.5 \%)$ other doctors.

With regard to managing children with acute mental health issues, $79 \%$ of registrars were involved at least weekly, with $85 \%$ of consultants involved monthly.

Only 3 doctors (11\%) had sectioned a child. Reasons for sectioning were suicidal ideation, food refusal, and acute mental health issues.
All staff surveyed had received support from CAMHS or the hospital crisis team. All of the doctors involved in sectioning children had found the situation difficult and stressful.

$78 \%$ of doctors surveyed felt that further training on sectioning patients should be available. Those respondents who said disagreed or were unsure about this gave the suggestion that this should be the responsibility of the CAMHS team. There were, however, concerns raised about the availability of urgent expert CAHMS assessment and advice.

Conclusions Children with mental health issues are increasingly presenting acutely to paediatric units. Paediatric registrars appear to be more frequently involved than consultants in their acute treatment. The experience of sectioning a child is rare and usually directed by CAMHS. This is recognised to be a difficult process for paediatricians, who have limited knowledge and confidence in this process.

This survey demonstrates that the vast majority of paediatricians would welcome further training and support in this area.

\section{G83(P) 'LEARNING FROM EXCELLENCE': EXPERIENCE IN A DISTRICT GENERAL HOSPITAL PAEDIATRIC DEPARTMENT}

K Jefferies, S Bell. Wexham Park Hospital, Frimley Health NHS Trust, Slough, UK

10.1136/archdischild-2018-rcpch.81

Aims Dr A.Plunkett pioneered the philosophy of 'learning from excellence'. The method was initiated to highlight that observing and reporting excellence within healthcare can provide new opportunities for learning and improve staff morale. Our aim was to initiate excellence reporting within our Paediatric Department, assess the uptake amongst staff and evaluate how staff members have found the process.

Methods In July 2017, excellence reporting was initiated. Reporting boxes were distributed within Paediatrics and Neonates. Paper forms are available in these clinical areas which include the report date, name of sender and receiver, reason for the report and details about how we can learn from this. Once a report has been submitted it's collected by a named Consultant who emails the sender and receiver with certificates. The receiver is also sent the written comments.

After initiation of the scheme, questionnaires were distributed to both senders and receivers of the reports to obtain feedback on the process.

Results In the first three months, 78 excellence reports were submitted. Nurses received reports most frequently (32\%), followed by trainees (28\%). Consultants, clerical staff, student nurses and play specialists have also been reported.

The most commonly reported themes included good team work (38\%), good clinical skills (16.7\%) and excellent communication skills $(11.5 \%)$.

Feedback from recipients revealed that $87.5 \%$ felt more appreciated at work after their report. $93.7 \%$ were very happy to have received their report and $100 \%$ felt receiving a certificate aswell as the written report worked well. $87.5 \%$ of recipients said that they were more inclined to report a colleague following their nomination and this has improved uptake within the department.

$100 \%$ of senders found the process of completing and submitting an excellence report easy. 
$100 \%$ of senders and recipients strongly agreed that the initiative was a good idea and should be continued.

Conclusion Current feedback is extremely positive. Morale within the department has improved and staff have been very receptive to the scheme. 'Learning from Excellence' has highlighted the importance of focusing on positive actions as well as errors and mistakes within the healthcare environment.

\section{G84(P) HOW APPROPRIATE ARE OUR EEGS?}

${ }^{1} \mathrm{G}$ Lanz, ${ }^{2} \mathrm{U}$ O'Neill, ${ }^{2} \mathrm{D}$ Cassidy. ${ }^{1}$ Medical School, Queens University Belfast, Belfast, UK; ${ }^{2}$ Paediatric Department, Daisy Hill Hospital, Southern Health and Social Care Trust, Newry, UK

\subsection{6/archdischild-2018-rcpch.82}

Aims An increase in EEG waiting times prompted an audit of EEG referrals, using the NICE guideline, CG137, to define our audit criteria. We aimed to audit all paediatric EEG referrals made from our paediatric service, between January to December 2016.

We aimed to audit the following:

1. Was the referral appropriate i.e. was it being carried out for suspected epilepsy, or inappropriate i.e. probable syncope or non-epileptic event.

2. Was the length of time between referral and EEG being performed within 4 weeks?

3. In the case of suspected epilepsy with a normal first EEG, was a further sleep deprived EEG (SDE) requested.

4. Patient diagnosis following investigation

Methods This was a retrospective audit, using information gathered from the Northern Ireland Electronic Care Record. The local EEG department supplied a list of all EEG referrals made in 2016 from our paediatric department. For each referral, we recorded the following: indication for referral, referral waiting time, EEG type and patient diagnosis. Using this information, we were able to categorise referrals as appropriate or inappropriate.

Results 131 patients were identified by the EEG department. We excluded 36 patients with known epilepsy and 4 patients who were under 3 months at the time of their seizure, leaving 91 patient records available for the audit. Of these, 5 patients were not counted; 1 patient's information was unavailable and 4 patients did not attend for EEG or follow-up appointments. A total of 86 patient records were included in the final audit. $60.5 \%$ of EEG referrals were assessed as appropriate. $39.5 \%$ were deemed inappropriate.

$29.8 \%$ of patients had an EEG carried out within 4 weeks. The average outpatient EEG waiting time was 66 days.

17 patients with suspected epilepsy with normal EEG, went on to have a SDE.

15 patients received a new diagnosis of epilepsy.

Conclusion Our results show that EEGs are being requested for suspected non-epileptic events which leads to the risk of false positive results, increased waiting times and unnecessary tests being carried out. We recommend that further education is required to support clinicians in requesting tests judiciously.

\section{G85(P) ADMISSIONS TO HOSPITAL AND AWARENESS OF KAWASAKI DISEASE IN ENGLAND}

${ }^{1}$ AM George, ${ }^{2}$ R McCormack, ${ }^{1,2}$ RMR Tulloh. 'Department of Paediatric Cardiology, Bristol Royal Hospital for Children, Bristol, UK; ${ }^{2}$ Societi, UK Foundation for Kawasaki Disease, Newark, UK

10.1136/archdischild-2018-rcpch.83

Background Kawasaki disease (KD) is now the commonest acquired heart disease in children in the Western world. Recent studies, such as the British Paediatric Surveillance Unit study, have suggested that the incidence in the UK is now around 9.1/100,000 children under 5 years. In May 2016, a national NHS England patient safety alert (PSA) was announced. Patients with coronary artery aneurysms (CAA) due to $\mathrm{KD}$ were to be alerted to the PSA, recalled and receive a patient specific protocol. Children in the UK have amongst the poorest outcomes globally with 24\% experiencing CAA despite treatment. We wished to determine if admissions with KD increased during the last 10 years.

Methods We undertook a Freedom of Information survey of all NHS Trusts in England (who would have received the PSA), to determine admissions with KD from 2005-2016. We asked whether the PSA had been acted upon and whether patients were recalled with CAA and aware of the PSA and which guidelines were being followed.

Results A poor response was seen to the PSA with only 11 Trusts intending to inform patients of the PSA, at routine clinic visits with no active recall. The PSA was circulated to clinicians through routine channels with few cascades to relevant clinicians. 81 Trusts reported admissions had increased four-fold from 130 per year to 400 per year, $63 \%$ being male. A further $10 \%$ of Trusts have yet to reply. Of those responding, under $50 \%$ were using the published guideline from 2013, most had a local guideline or were using no guideline. Over the same period, presenting age reduced from median 2.8 years to median 1.6 years.

Conclusions There is a marked increase in admissions in the UK coded as KD over the 10 years from 2006-2015. Despite this, few Trusts have responded to the PSA and awareness of the disease and its complication rate amongst the medical community and general public remains low. Significantly increasing incidence and poor levels of awareness have resulted in late diagnosis beyond the recommended 5 days of illness, resulting in increased risks of CAA with expected long term effects.

\section{G86(P) BIOMARKERS FOR CORONARY ARTERY ANEURYSMS IN KAWASAKI DISEASE}

${ }^{1}$ AM George, ${ }^{1,2}$ RMR Tulloh. ${ }^{1}$ University of Bristol, Bristol, UK; ${ }^{2}$ Bristol Royal Hospital for Children, Bristol, UK

10.1136/archdischild-2018-rcpch.84

Background Kawasaki disease (KD) is now the commonest acquired heart disease in the Western world estimated in the UK at 9.1/100,000 children under 5 years. 24\% of children in the UK develop coronary artery aneurysms (CAA) despite 